\title{
High harmonic generation with long-wavelength few-cycle laser pulses
}

\author{
Bruno E Schmidt ${ }^{1}$, Andrew D Shiner ${ }^{2}$, Mathieu Giguère ${ }^{1}$, \\ Philippe Lassonde $^{1}$, Carlos A Trallero-Herrero ${ }^{3}$, J-C Kieffer ${ }^{1}$, \\ P B Corkum ${ }^{2}$, D M Villeneuve ${ }^{2}$ and François Légaré ${ }^{1}$
}

\author{
${ }^{1}$ Institut National de la Recherche Scientifique, Centre Énergie Matériaux et Télécommunications, \\ 1650 Boulevard Lionel-Boulet, Varennes, QC J3X1S2, Canada, \\ ${ }^{2}$ Joint Laboratory for Atto-Second Science, University of Ottawa/NRC, 100 Sussex Drive, Ottawa, \\ ON K1A 0R6, Canada \\ ${ }^{3}$ J. R. Macdonald Laboratory, Physics Department, Kansas State University, Manhattan, KS 66506, USA \\ E-mail: schmidtb@emt.inrs.ca and legare@emt.inrs.ca
}

Received 15 September 2011, in final form 10 November 2011

Published 16 March 2012

Online at stacks.iop.org/JPhysB/45/074008

\begin{abstract}
We report the extension of hollow-core fibre pulse compression to longer wavelengths.

High-energy multi-cycle infrared pulses are generated via optical parametric amplification and subsequently broadened in the fibre. 2.5 -cycle pulses at the Signal wavelength $(1.4 \mu \mathrm{m})$ and 1.6-cycle pulses at the Idler wavelength $(1.8 \mu \mathrm{m})$ in the sub-millijoule regime have been generated. New compression schemes can be applied at $1.8 \mu \mathrm{m}$ and beyond. In this manner, 1.6-cycle carrier envelope phase stable pulses were generated by linear propagation in the anomalous dispersion regime of bulk glass which surprisingly enables compression below its third-order dispersion limit. Furthermore, a dispersion-free way of controlling the carrier envelope phase is demonstrated. Moreover, we experimentally confirm the increase in high-harmonic cut-off energy with driving laser wavelength and demonstrate the beneficial effect of few-cycle pulses which enable higher saturation intensities on target compared to multi-cycle pulses. It will be an ideal tool for future synthesis of isolated attosecond pulses in the sub-keV regime. With this laser source, we revealed for the first time multi-electron effects in high harmonic generation in xenon.
\end{abstract}

(Some figures in this article are in colour only in the electronic version)

\section{Introduction}

In 1987, Fork et al achieved a long-lasting world record of pulse compression down to $6 \mathrm{fs}$ at $\sim 620 \mathrm{~nm}$ wavelength based on spectral broadening through nonlinear propagation of $50 \mathrm{fs}$ laser pulses in a single-mode fibre (SMF) followed by phase compensation up to cubic order with a combination of prism and grating compressors [1]. This unique laser source rapidly paved the way for a variety of time-resolved spectroscopy experiments requiring high temporal resolution [2-5]. Limited to a few nanojoules of energy per pulse due to the SMF, those pulses were not intense enough to study strong field processes such as high harmonic generation (HHG).

In 1996, Nisoli et al extended the approach of spectrally broadening multi-cycle pulses to the millijoule energy level of high-power Ti:Sa chirped pulse amplifiers. They replaced the SMF by a hollow-core fibre (HCF) filled with gas as the nonlinear medium [6]. Dispersion compensation with a prism pair subsequent to the HCF enabled generation of $10 \mathrm{fs}$ pulses at $0.8 \mu \mathrm{m}$ centre wavelength. By the end of the $1990 \mathrm{~s}$, generation of intense $5 \mathrm{fs}$ pulses at Ti-Sa wavelength became possible due to invention of chirped mirrors (CM). Meanwhile, strategies to stabilize the carrier envelop phase (CEP) of laser pulses were developed $[7,8]$ leading to the first demonstration of isolated attosecond pulses generated by the process of HHG in gas medium in 2001 [9]. Nowadays, attosecond metrology is used to probe the fastest electronic processes in atoms [10-12], molecules [13] and condensed matters [14]. Recent advancement in CM technology enabled dispersion compensation over an octave of bandwidth providing 
single-cycle Ti-Sa laser pulses [15] which were used to generate an 80 attosecond pulse duration in the sub-100 eV spectral range [16].

Obtaining even shorter x-ray pulses via HHG requires extending attosecond metrology to higher photon energies and larger spectral bandwidth [17]. Higher photon energies have been demonstrated with laser sources delivering longer wavelengths compared to $\mathrm{Ti}-\mathrm{Sa}[18-21]$. This can be understood by the three-step model as described by Corkum in 1993 [22], which predicts the cut-off photon energy $\left(E_{\mathrm{C}}\right)$ of the HHG spectra to be given by $E_{\mathrm{C}}(\mathrm{eV})=I_{\mathrm{P}}+3.17 U_{\mathrm{P}}$ (cutoff law), with $I_{\mathrm{P}}$ being the ionization potential of the atom and $U_{\mathrm{P}} \sim I \lambda^{2}$. Increasing laser intensity $(I)$ on target, however, must not exceed the threshold for depletion of population in the neutral state which strongly depends on $I_{\mathrm{P}}$. One approach to overcome this limitation of generating higher cut-off energy is to increase the driving laser wavelength. While several research groups have shown cut-off extension with longer laser wavelength [18-21], isolated attosecond pulses have not yet been demonstrated at photon energies higher than $110 \mathrm{eV}$. With the aim of transferring established strategies for the generation of isolated attosecond pulses from $0.8 \mu \mathrm{m}$ to the IR spectral range, a few research groups have been working to develop laser sources delivering CEP stabilized few-cycle pulses at longer wavelength.

Different approaches have been investigated to generate $\mathrm{mJ}$ level CEP stabilized few-cycle laser pulses. They are (1) the optical parametric chirped-pulse amplifier providing sub$\mathrm{mJ}$ laser pulses at $2.1 \mu \mathrm{m}[23,24]$, pulse self-compression by filamentation at $\sim 2 \mu \mathrm{m}[25,26]$ and the generation of CEP stabilized $1.5 \mu \mathrm{m}$ few-cycle laser pulses by difference frequency generation (DFG) of ultra-broadband $0.8 \mu \mathrm{m}$ laser pulses followed by broadband parametric amplification [27]. In this paper, we show that spectral broadening in an argonfilled HCF followed by a proper dispersion compensation scheme (CM at $1.4 \mu \mathrm{m}$ and a fused silica window at $1.8 \mu \mathrm{m}$ [28]) is a robust and simple approach to obtain few-cycle IR pulses. It can be extended to even longer wavelengths, as long as glasses are not absorbing [29]. In addition, starting with a white light seeded optical parametric amplifier provides passive CEP stabilization [30].

Non-CEP stabilized pulses have been applied to perform HHG in xenon described in [31]. Here we expand on these results, showing the spectral evolution of HHG from xenon as a function of wavelength and laser pulse duration. We verify the extension of the XUV spectrum with longer laser wavelength according to the cut-off law. Furthermore, we demonstrate how few-cycle pulses allow higher intensities on target before depletion of ionization of the neutral ground state compared to longer pulse durations. This new laser technology enabled our research team to probe the giant resonance of xenon in the proximity of $100 \mathrm{eV}$ and to extend the cut-off up to $160 \mathrm{eV}$.

\section{Experimental method}

The following experiments have been performed on two different laser systems at the advanced laser light source in Varennes, Canada. They share the common principle

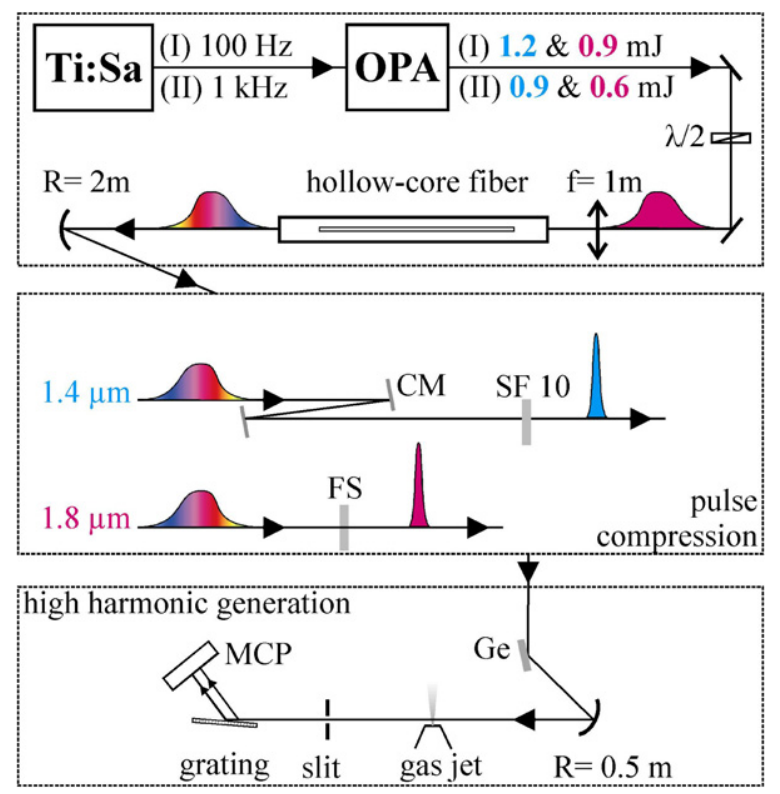

Figure 1. General sketch of the experimental setup representing two different laser systems (I) and (II). The upper panel shows IR pulse generation via commercial OPA systems pumped by a Ti-Sa amplifier. A plano-convex lens couples the light into a standard hollow-core fibre for spectral broadening in an argon atmosphere. Signal pulses are compressed with chirped mirrors (CM), whereas Idler pulses are compressed only with an FS window to sub-two cycle duration. Blue is chosen for Signal wavelengths, whereas red represents Idler pulses in the middle panel. Those pulses are focused in a thin gas jet of xenon and the resulting XUV light from $\mathrm{HHG}$ is recorded with an imaging spectrometer and a micro channel plate (MCP, lower panel).

of starting from narrowband, high-energy IR laser pulses generated via type II amplification with a commercially available OPA. After that, the OPA output is spectrally broadened in an HCF to achieve the large bandwidth required for few-cycle pulse generation, as shown in the upper panel of figure 1. The fact that we performed pulse compression experiments with two different Ti:Sa laser systems which are not CEP stabilized underlines the universality of our approach. Each Ti:Sa amplifier is pumping a different type of highenergy OPA. Firstly, we worked with a $100 \mathrm{~Hz}, 12 \mathrm{~mJ}$, 45 fs laser system (Thales) from which $6 \mathrm{~mJ}$ was split to pump a superfluorescence seeded high-energy OPA (Light Conversion, HE-TOPAS, labelled (I) in figure 1). Secondly, a $1 \mathrm{kHz}, 3.8 \mathrm{~mJ}, 35 \mathrm{fs}$ Ti-Sa laser (KMLabs) was used in combination with a white light seeded OPA likewise from Light Conversion (labelled (II) in figure 1). The advantage of using a white light rather than fluorescence seeded OPA lies in the passive CEP stabilization of Idler pulses in the first case [32].

In the last stage of both OPAs, pumping is performed at a slight angle $\left(<1^{\circ}\right)$ with respect to the seed in order to separate Signal and Idler beams spatially after some distance. In all cases, coupling into the $\mathrm{HCF}$ is carried out with a $1 \mathrm{~m}$ plano-convex lens made of $\mathrm{CaF}_{2}$, a material also used for all other windows in the setup due to its small amount of dispersion in the 1-2 $\mu \mathrm{m}$ spectral range. The HCF length is 
$1 \mathrm{~m}$ with an inner diameter of $400 \mu \mathrm{m}$ which is filled with argon for spectral broadening. Pressure of argon was adjusted to control the spectral width at the HCF output and to minimize instabilities.

For both laser systems $(100 \mathrm{~Hz}$ and $1 \mathrm{kHz})$, Signal and Idler wavelengths were compressed in different ways as will be explained in the following. Signal pulses around $1.4 \mu \mathrm{m}$ were compressed with one round trip on CMs which were designed in collaboration with Femtolasers [33]. They introduce negative group delay dispersion (GDD) to compensate for the primarily quadratic chirp stemming from self-phase modulation (SPM) in the HCF, as well as small, negative third-order dispersion (TOD) to balance positive TOD of glasses eventually used in the setup [33].

Different to this established pulse compression method with CM which is sketched in the middle panel of figure 1, we established a new scheme for compression of Idler fewcycle pulses around a central wavelength of $1.8 \mu \mathrm{m}$. It simply utilizes linear propagation through a single window of FS instead of CM to balance the spectral phase introduced in the HCF. At a first glance, this seems very straightforward. Since many glasses exhibit anomalous dispersion in the IR range they provide negative GDD to cancel the major part of the spectral phase arising from SPM. However, all dielectric media provide positive TOD throughout their transparent spectral range. To our surprise, the experiment shows that linear propagation in bulk material after the HCF can lead to pulse compression below its TOD limit (TOD limit means full compensation of GDD only). This can be explained by the fact that in addition to SPM in the HCF also self-steepening of the pulse trailing edge occurs during nonlinear propagation. Assuming a symmetric temporal shape of the input pulse, pure SPM causes an intensity-induced symmetric variation of the pulse temporal phase without changes to the pulse envelope. This, in turn, causes a symmetric broadening of the power spectrum with respect to the OPA's fundamental wavelength as well as a symmetric spectral phase which can be approximated by a parabola centred around the fundamental. On the other hand, self-steepening imprints a temporal reshaping of both the pulse envelope and temporal phase which become asymmetric. In the spectral domain, this behaviour translates to an asymmetric power spectrum and what is essential for the new compression scheme, also to an asymmetry of the spectral phase. This additional phase asymmetry on top of the symmetric SPM is nicely opposed by the TOD of bulk material. This intuitive physical explanation was verified by numerical modelling of nonlinear propagation in the $\mathrm{HCF}$ and linear propagation in FS afterwards [29]. It is worth mentioning that self-steepening results from a nonlinear polarization of the argon gas without the need for plasma generation. Furthermore, this compression scheme was numerically proven to support clean single cycle pulses and experimentally quasi-single-cycle pulses of about 1.5 cycles have been demonstrated [30, 34].

Pulse characterization was carried out with a home-built second harmonic generation-frequency resolved optical gating
(SHG-FROG) setup ${ }^{4}$ and more details can be found in the literature $[28,33]$.

Subsequent to dispersion compensation, the few-cycle IR pulses were applied to study HHG at different wavelengths and with different pulse durations. A schematic diagram of the HHG spectrometer and source chamber is shown in the lower panel of figure 1. Before entering the vacuum chamber, an arrangement for variable attenuation over the Signal and Idler spectral range from 1.1 to $2.3 \mu \mathrm{m}$ has been installed. It enables energy control without changing the optical path after the HCF. This is realized by placing an achromatic half wave plate prior to the HCF whose spectral range is sufficient to cover the range from 1.4 to $1.8 \mu \mathrm{m}$. Depending on its rotation of the laser polarization, a corresponding fraction of light is reflected off the Germanium window (Ge in the lower panel of figure 1) which is mounted at Brewster's angle. Nonlinear propagation in the HCF is not affected by rotation of polarization.

The beam is then focused inside the vacuum chamber into a thin pulsed jet of xenon with an $R=0.5 \mathrm{~m}$ spherical silver mirror. The jet has a $500 \mu \mathrm{m}$ aperture; thus, it is much thinner than the Rayleigh range of the optical beam. It is typically operated at a backing pressure of 2-3 atm leading to an estimated number density of gas atoms in the jet of around $2 \times 10^{17} \mathrm{~cm}^{-3}$. The intensity was determined by varying the input pulse energy and measuring the amount of ionization in the gas jet. Ions were detected with a negatively biased wire mesh (not shown in figure 1) a few centimetres away from the focus. By fitting the measured ion signal versus input energy to the calculated ion signal based on the Yudin-Ivanov ionization model [35], the laser intensity can be determined [36]. This procedure is described in more detail in [28, 35].

The XUV light passes from the source to the detector chamber via a differential pumping tube after which the XUV beam enters the spectrometer through an $\sim 140 \mu \mathrm{m}$ slit. The XUV spectrum is dispersed by a $12001 \mathrm{~mm}^{-1}$ spherical holographic grating (Shimadzu) which was chosen because of its significantly lower second-order diffraction compared to equivalent ruled gratings $[37,38]$. The dispersed spectrum is imaged onto a microchannel plate (MCP) intensifier backed by a phosphor screen (Burle) and the spectrum is digitized with a high dynamic range digital camera (PCO).

\section{Experimental results and discussions}

As mentioned above, pulse compression has been investigated at 1.4 and $1.8 \mu \mathrm{m}$. Firstly, we used a parametric superfluorescence seeded OPA pumped by a $100 \mathrm{~Hz}$ Ti-Sa laser system and those results are presented in section 3.1. The same scheme was realized with a different Ti-Sa laser $(1 \mathrm{kHz})$, pumping a white light seeded OPA for which the results are presented in section 3.2. In both cases, the OPAs were tuned to generate a set of conjugated Signal and Idler pulses roughly at the same wavelengths to provide comparable conditions. This set of wavelengths was chosen because both OPAs show the highest output power and best stability. Section 3.3 describes

\footnotetext{
4 We employed geometrical beam splitting with a half mirror and used a thin type I BBO crystal of $10 \mu \mathrm{m}$ thickness. Vacuum windows, etc were accounted
} for in the FROG measurement. 

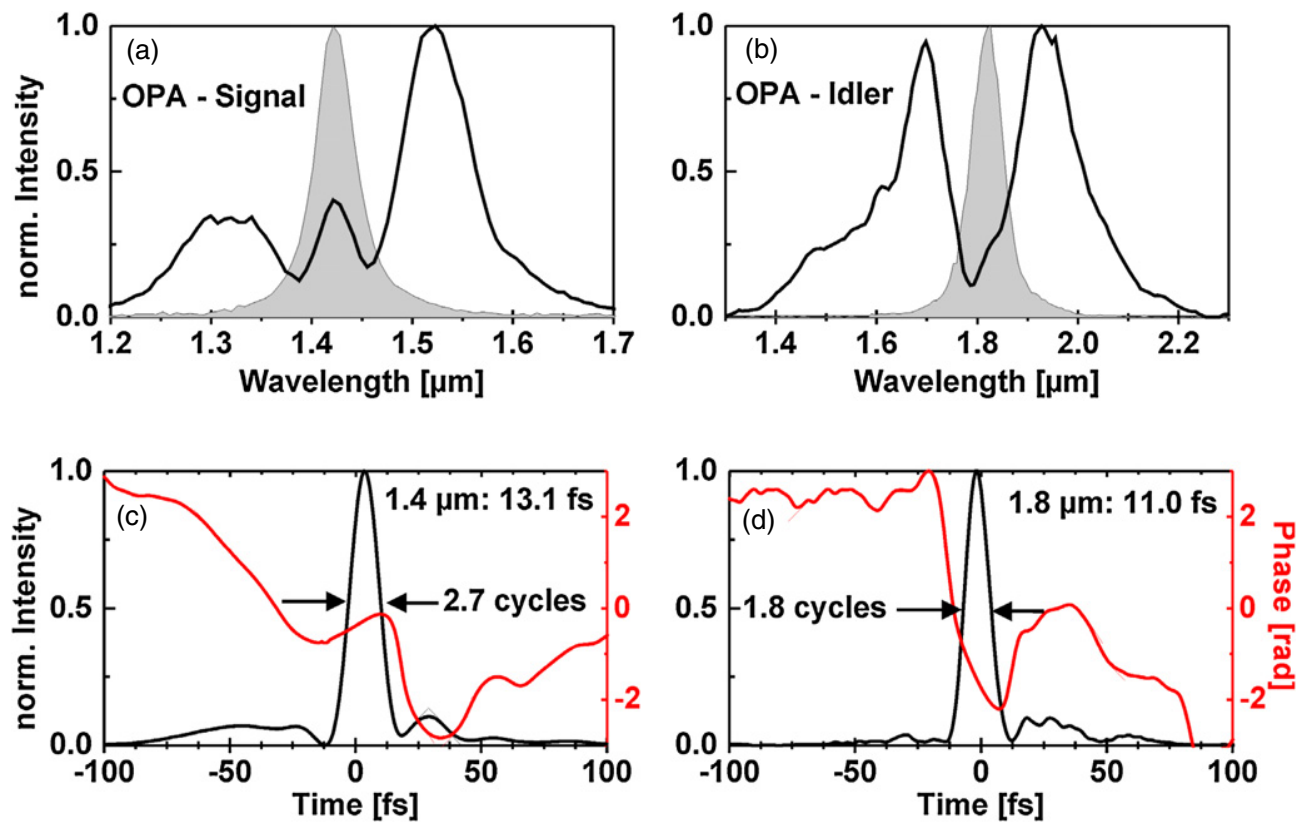

Figure 2. Results with superfluorescence seeded OPA. OPA output pulses (shaded grey) and after broadening in the hollow-core fibre (black) for (a) the Signal at $1.425 \mu \mathrm{m}$ wavelength and (b) the Idler at $1.82 \mu \mathrm{m}$ wavelength. Retrieved temporal intensity (black) and phase (red) for (c) Signal pulse compression with chirped mirrors and (d) Idler pulses after linear propagation through FS only.

a novel scheme for controlling the CEP in a white light seeded OPA.

Finally, the $100 \mathrm{~Hz}$ setup of section 3.1 was used for HHG in xenon and XUV spectra for different wavelengths and pulse durations of the driving laser are presented in section 3.4. Thanks to the long-wavelength few-cycle pulses, the XUV spectrum for low $I_{\mathrm{P}}$ species like xenon extends far enough enabling us to observe for the first time multi-electron correlation effects in xenon, in particular the giant resonance around $100 \mathrm{eV}$ photon energy.

\subsection{Superfluorescence seeded OPA (case I)}

The $100 \mathrm{~Hz}$ setup, case (I) in figure 1, employing a superfluorescence seeded OPA delivers Signal and Idler pulses whose energies are $1.2 \mathrm{~mJ}$ and $0.9 \mathrm{~mJ}$, respectively. Corresponding OPA spectra are shown in figures 2(a) and (b) as shaded grey curves. Those spectra are associated with pulse durations in the range of 50-60 fs. Pulse durations remained unchanged after the HCF. Effects of pulse self-shortening were not observed since the input power in each case was well below the critical power for self-focusing. Fibre output spectra are shown as black curves in figures 2(a) and (b), each normalized by their maximum value.

A transmission of $40 \%$ was achieved for the Signal pulses leading to $0.4 \mathrm{~mJ}$ output energy after the HCF. After passing the CM, SHG-FROG traces were recorded for different pressures of argon and with different SF10 windows to finetune dispersion. In this way, the shortest duration of $13.1 \mathrm{fs}$ was achieved with 1.7 bar of argon. The SHG-FROG retrieved temporal intensity is plotted in figure 2(c) in black as well as the temporal phase in red.

Now, we present results achieved with the OPA Idler on the $100 \mathrm{~Hz}$ laser system. Coupling into the HCF resulted in a transmission of about $50 \%$, slightly higher than in the Signal case with the same HCF. We attribute this to improvement mode quality of the Idler beam. Up to $0.45 \mathrm{~mJ}$ pulse energy was obtained after the fibre with a duration of about $50 \mathrm{fs}$ and the broadened spectrum for 1.2 bar of argon is given in figure 2(b). An equivalent of about $4 \mathrm{~mm}$ FS was used to generate $11 \mathrm{fs}$ pulses at $1.8 \mu \mathrm{m}$ wavelength. This denotes about $36 \mathrm{GW}$ of pulse power within 1.8 optical cycles FWHM, an ideal source to study strong field processes such as HHG. A rather clean temporal shape is visible in the plot of figure 2(d) which is carrying about $77 \%$ of total energy in the main pulse.

\subsection{White light seeded OPA (case II)}

Now we switch to the second Ti-Sa laser system. Besides increasing the repetition rate to $1 \mathrm{kHz}$, it is capable of delivering shorter pulses at $0.8 \mu \mathrm{m}$ as compared to the $100 \mathrm{~Hz}$ system. The white light seeded OPA generates Signal and Idler pulses with energies of 0.9 and $0.6 \mathrm{~mJ}$, respectively. Although the last amplification crystal in both high-energy TOPAS, superfluorescence and white light seeded, has the same cut and thickness, the spectra for the latter are slightly broader, as can be seen when comparing the shaded grey curves of figures 3(a) and (b) and 2(a) and (b). In accordance with the broader spectra, the OPA pulses were slightly shorter in the range of 30-40 fs.

Spectral broadening of Signal pulses in 2.3 bar of argon leads to the continuum shown in figure 3(a) as a black curve. Since the energy directly in front of the HCF was $0.8 \mathrm{~mJ}$ and we obtained a transmission of about $42 \%$, we measured $0.35 \mathrm{~mJ}$ pulse energy after the HCF. The same CM setup as described in section 3.1 leads to pulse compression down to $12.1 \mathrm{fs}$, shown in figure 3(c) as a black curve. The shorter duration of $12.1 \mathrm{fs}$ 

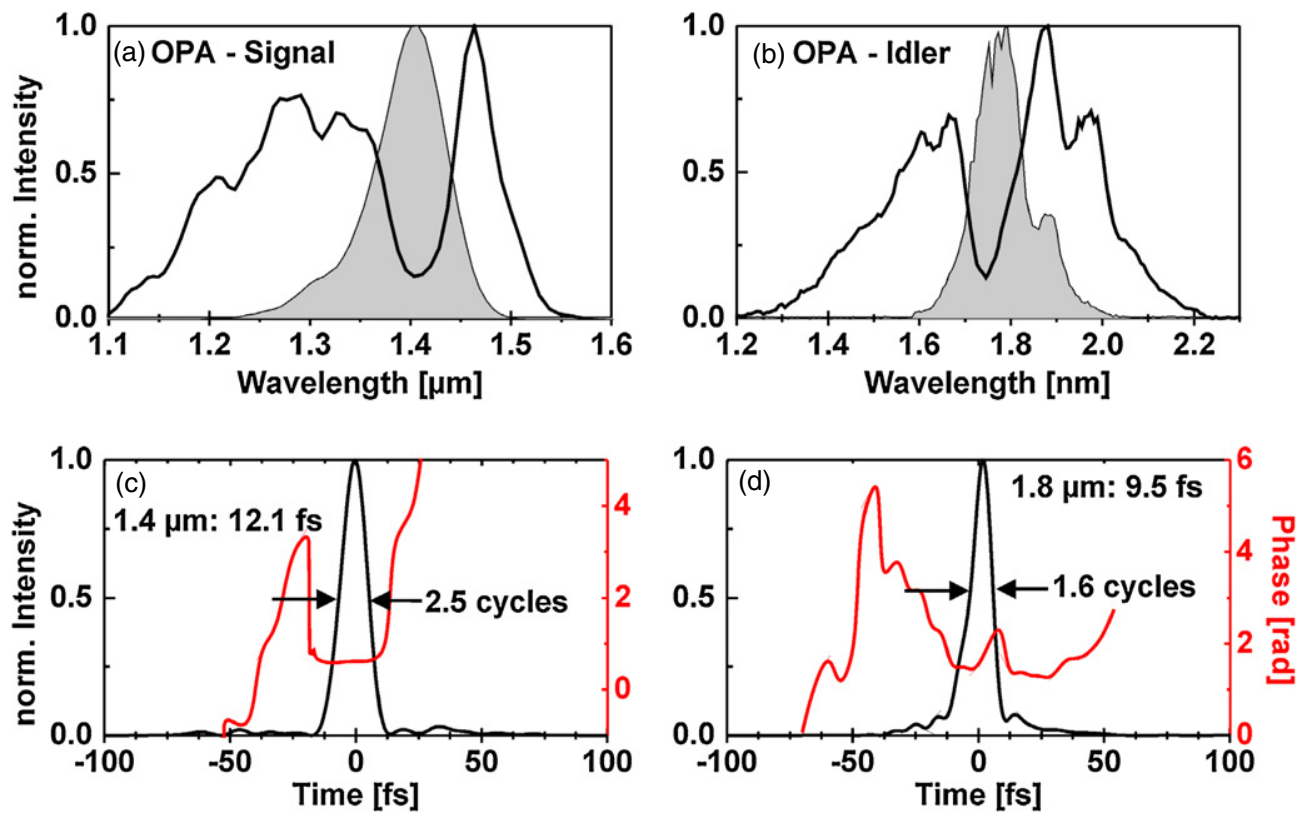

Figure 3. White light seeded OPA results. Output spectra of OPA (shaded grey) and after the hollow-core fibre (black) for (a) the Signal at $1.4 \mu \mathrm{m}$ wavelength and (b) the Idler at $1.78 \mu \mathrm{m}$ wavelength. Retrieved temporal intensity (black) and phase (red) for (c) Signal pulse compression with chirped mirrors, and (d) Idler pulses after linear propagation through FS only.

compared to $13.1 \mathrm{fs}$ in section 3.1 is linked to the shorter input duration coming from the white light seeded OPA which was 42 fs compared to $60 \mathrm{fs}$ from the superfluorescence seeded one.

From a physical point of view, it is obvious that shorter input pulses require less SPM to achieve the same spectral broadening as longer input pulses. The broad spectrum in figure 3(a) exhibits only one minimum in the supercontinuum whereas the one of figure 2(a) shows two minima and a third peak arises at the central wavelength. From simulations, it can be seen that stronger SPM leading to a three peaked continuum is also accompanied by a more complex spectral phase function which shows an increasing number of oscillations around the central wavelength limiting the potential for clean pulse compression.

The same arguments hold for the Idler bulk pulse compression scheme for which the input pulse duration of $35 \mathrm{fs}$ was shorter on the $1 \mathrm{kHz}$ system compared to the situation in section 3.1. The input spectrum before the HCF is shown in figure 3(b) as a shaded grey curve and spectral broadening in 2.3 bar argon is given by a black curve. After the fibre, linear propagation through $1.5 \mathrm{~mm}$ of FS suitably compresses the spectral phase to 9.5 fs FWHM duration of intensity. This corresponds to 1.6 optical cycles at $1.8 \mu \mathrm{m}$ wavelength.

The transform-limited (TL) duration for the spectrum in figure 3(b) is 9.2 fs and the residual uncompensated higher order spectral phase is responsible for observed deviation from the theoretical value. Although pedestals arise when aiming for the shortest FWHM duration, the main peak still carries $83 \%$ of the total pulse power. To emphasize the high fidelity of phase compensation with bulk material we calculated the effect of uncompensated TOD on few-cycle pulse compression. Assuming perfect cancellation of the GDD component, solely the travel through $1.5 \mathrm{~mm}$ FS would broaden a TL $9.5 \mathrm{fs}$ pulse to $11.9 \mathrm{fs}$.

Another important attribute of the 1.6-cycle pulse of figure 3(d) is its passive CEP stability which was confirmed with an $f-2 f$ interferometer. To build this, no additional broadening stage had to be implemented to generate the octave spanning spectra since the blue spectral wing in figure 3(b) extends out to $0.7 \mu \mathrm{m}$ which cannot be seen in the linear plot. Thus, only a type I BBO for SHG of IR and a polarizer to project both the broadband spectrum and its doubled fundamental is required to perform $f-2 f$ measurements. An rms stability of $350 \mathrm{mrad}$ was characterized in this manner [30] on the $1 \mathrm{kHz}$ laser system.

\subsection{CEP control}

Very recently, the $100 \mathrm{~Hz}$ laser system was upgraded with a white light seeded OPA yielding pulse durations of 1.9 optical cycles which are passively stabilized [32]. The passive CEP stabilization stems from DFG between a white light continuum ( $\left.\Phi_{\text {white light }}\right)$ and a remaining portion of the $800 \mathrm{~nm}$ pump beam $\left(\Phi_{\text {pump }}\right)$, both sharing the same randomly fluctuating CEP. Since the DFG process conserves not only the quantities of energy and momentum but also the phases, the CEP of the Idler $\left(\Phi_{\text {Idler }}\right)$ is constant:

$$
\Phi_{\text {Idler }}=\Phi_{\text {pump }}-\Phi_{\text {white light }}-\Phi / 2=\text { const. }
$$

From equation (1) it can be seen that an additional constant phase offset added to $\Phi_{\text {pump }}$ or $\Phi_{\text {white light }}$ transfers to a phase shift of the Idler pulse. We implemented this phase shifter in the white light arm of the OPA by moving a steering mirror with a piezoelectric actuator [39]. We carefully checked that the mirror movement on the order of the wavelength does neither affect the few-cycle pulse compression nor the CEP 


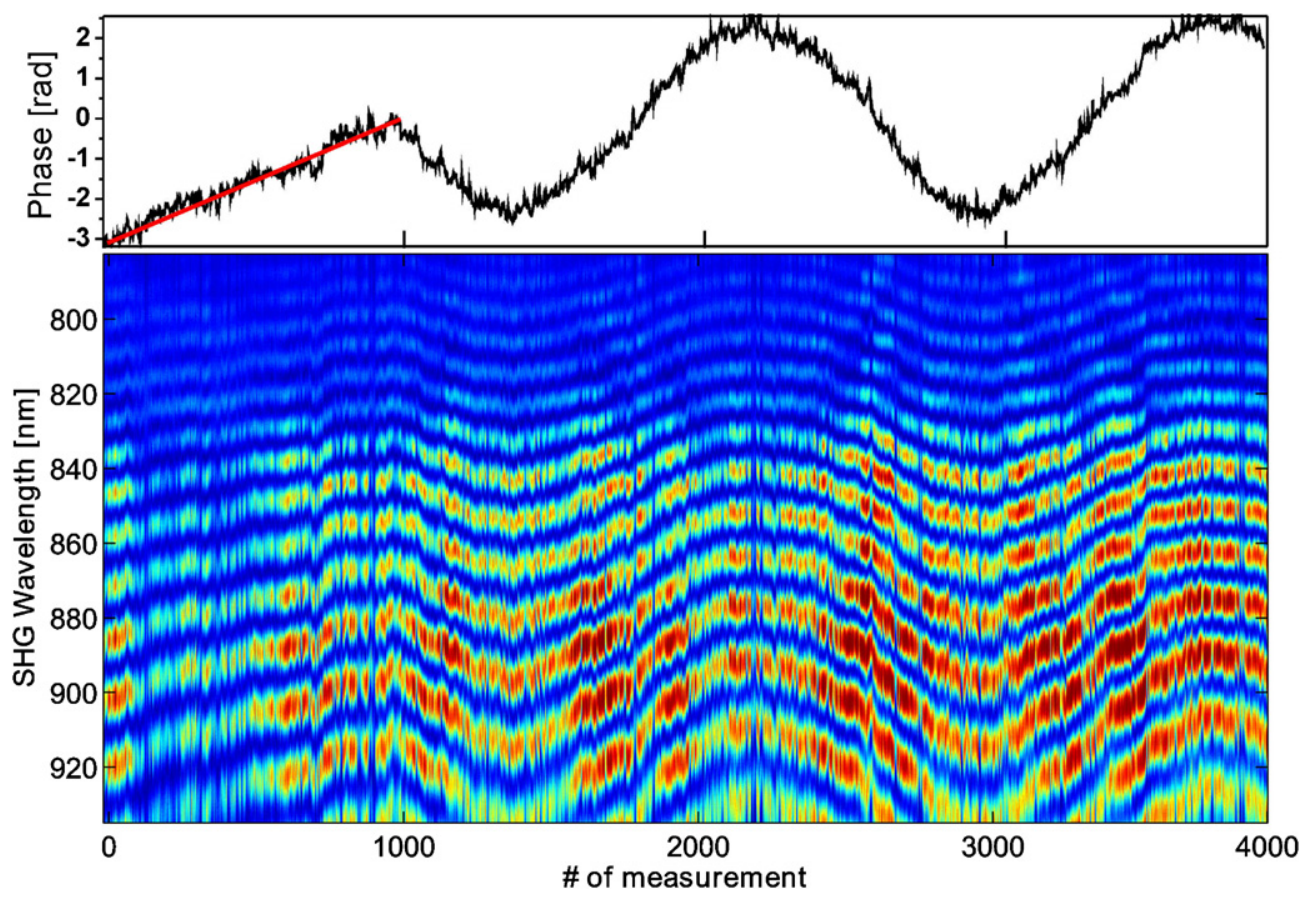

Figure 4. Passive CEP stabilization and control without a feedback loop over an acquisition time of 6.7 min. A linear phase ramp is applied for the first thousand samples and a sinusoidal modulation for the lasting part.

stability. This approach for CEP control is fast, dispersion free and fully wavelength and bandwidth independent since it circumvents changing material dispersion as done in many experiments [7].

Figure 4 demonstrates the passive CEP stabilization and CEP control without a feedback loop for a set of 4000 consecutive $\mathrm{f}-2 \mathrm{f}$ interferograms in the lower panel. Each interferogram is averaged over ten laser shots denoting a total acquisition time of $6.7 \mathrm{~min}$. The value for the relative CEP is shown in the upper graph of figure 4. For the first thousand measurements, the CEP was continuously ramped from $-\pi$ to 0 and a sinusoidal modulation was applied for the rest of the CEP scan. In the region of the CEP linear slope (red line in the upper panel), an rms stability of $410 \mathrm{mrad}$ was evaluated based on 10-shot averaging. To reduce this value we will investigate the influence of beam pointing fluctuations when coupling into the fibre as well as from unwanted ionization stemming from residual air inside the $\mathrm{HCF}$ vacuum assembly.

\subsection{HHG in xenon using few-cycle IR laser pulses}

In this section, the application of few-cycle IR pulses for HHG is presented. In particular, we compare HHG spectra obtained at different wavelengths as well as different pulse durations. We utilized the $100 \mathrm{~Hz}$, non-CEP stabilized, superfluorescence seeded OPA for HHG and the results are shown in figure 5. Spectra attained with Signal and Idler pulses, respectively, are linked to bluish and reddish colors. The thin black curve shows HHG with a long pulse of 63 fs at $800 \mathrm{~nm}$ wavelength at an intensity of $8.8 \times 10^{13} \mathrm{~W} \mathrm{~cm}^{-2}$. All intensity values are calculated by fitting ionization data according to the Yudin-Ivanov ionization model [35] as described in section 2. This has the advantage of determining the intensity in the actual interaction region regardless of focusing conditions and positioning of the focus relative to the gas jet.

An important aspect is to define conditions under which it is possible to compare HHG spectra taken at very different wavelengths $(0.8-1.8 \mu \mathrm{m})$ and different pulse durations (231.8 cycles). Therefore, we present $\mathrm{HHG}$ spectra recorded close to the corresponding saturation intensity for each individual condition. The saturation intensity is defined when $43 \%$ of the neutral ground-state population of the gas is ionized by the most intense part of the laser pulse on axis.

Furthermore, all plots of figure 5 are normalized to their corresponding maximum at about $20 \mathrm{eV}$. The aim of this comparison, however, is not to evaluate the absolute efficiency of HHG under different conditions but to demonstrate the cut-off extension when (i) moving to longer wavelengths and (ii) using few-cycle pulses. All spectra are averaged over hundreds of laser shots to achieve a good signal-to-noise ratio in the cut-off region. The spectrum plotted as a blue curve in figure 5 is measured with a 12-cycle pulse (55 fs) at $1.4 \mu \mathrm{m}$. It shows a comb-shaped XUV spectrum up to the cut-off at about $65 \mathrm{eV}$, typical for HHG with multicycle pulses due to spectral interferences occurring from many half-cycle emissions. Reducing the Signal pulse duration to 3.2 cycles (15 fs at $1.425 \mu \mathrm{m}$ ) yields the cyan curve of figure 5 which is touching the $90 \mathrm{eV}$ margin. The spectrum becomes smooth beyond $60 \mathrm{eV}$. It agrees with the picture of a reduced modulation contrast in the HHG spectrum since XUV emission is restricted to less and less half optical cycles within the pulse envelope. Moreover, the decreased modulation contrast is not due to reduced spectrometer resolution as can be seen when compared with the HHG spectrum obtained with the multi-cycle pulses at $1.82 \mu \mathrm{m}$, depicted as the red curve. For an equal number of 12 cycles ( $73 \mathrm{fs}$ at $1.8 \mu \mathrm{m}$ ) for the Idler 


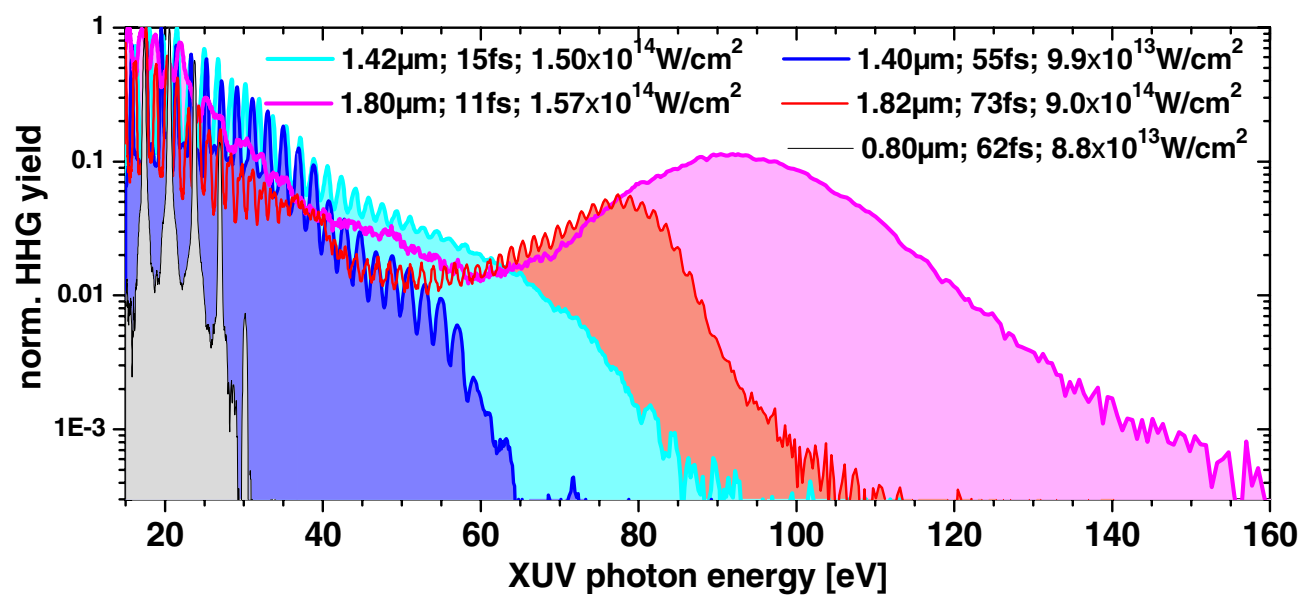

Figure 5. Semi-log plot of high harmonic yield in xenon at different driving laser wavelengths (grey: $0.8 \mu \mathrm{m}$; bluish: $1.4 \mu \mathrm{m}$; reddish: $1.8 \mu \mathrm{m})$. Each measurement was performed at intensity close to the corresponding saturation intensity and shows the benefit of increased driving wavelength and decreased pulse duration, respectively. Pulse durations and intensities are given in the legend. The striking enhancement around $95 \mathrm{eV}$ originates from the giant resonance in xenon, an effect due to multi-electron correlation upon the recombination step in HHG.

Table 1. Comparison of conditions (duration, intensity) under which HHG was driven at three different wavelengths.

\begin{tabular}{llllll}
\hline $\begin{array}{l}\text { Wavelength } \\
(\mu \mathrm{m})\end{array}$ & $\begin{array}{l}\text { Duration } \\
\text { (number of } \\
\text { cycles) }\end{array}$ & $\begin{array}{l}\text { Saturation } \\
\text { intensity } \\
\left(10^{14} \mathrm{~W} \mathrm{~cm}^{-2}\right)\end{array}$ & $\begin{array}{l}\text { Experimental } \\
\text { intensity } \\
\left(10^{14} \mathrm{~W} \mathrm{~cm}^{-2}\right)\end{array}$ & $\begin{array}{l}\text { Calculated } \\
\text { cut-off } \\
(\mathrm{eV})\end{array}$ & $\begin{array}{l}\text { Measured } \\
\text { cut-off } \\
(\mathrm{eV})\end{array}$ \\
\hline 0.8 & 23 (long) & 0.84 & 0.88 & 28.5 & 30 \\
1.4 & 12 (long) & 0.99 & 0.99 & 70 & 65 \\
& 3.2 (short) & 1.37 & 1.5 & 102 & 90 \\
1.8 & 12 (long) & 0.98 & 0.90 & 100 & 105 \\
& 1.8 (short) & 1.52 & 1.57 & 166 & 160 \\
\hline
\end{tabular}

pulse, a clearly modulated spectrum extends out to $105 \mathrm{eV}$ (red shaded curve). Table 1 summarizes the experimental finding of figure 7. Measured and calculated cut-off photon energies are in good agreement from 30 to $160 \mathrm{eV}$ for all laser wavelengths and pulse durations.

Another conspicuous feature of the red curve is the fact that harmonic yield increases at the cut-off region. It rises from 55 to $80 \mathrm{eV}$ before it starts to roll off in contrast to the blue curves for the OPA Signal pulses which only decrease monotonically. This increase continues even further when HHG is driven by the 1.8 -cycle pulse (11 fs) at $1.8 \mu \mathrm{m}$. The striking enhancement at $95 \mathrm{eV}$ in figure 5 is attributed to the giant resonance in xenon, which was first observed in photoionization measurements at synchrotron sources [40]. The enhancement originates from the $4 \mathrm{~d}$ sub-shell $(\mathrm{Xe}=[\mathrm{Kr}]$ $4 d^{10} 5 s^{2} 5 p^{6}$ ) and was explained theoretically by the combined effects of a shape resonance as well as collective interactions between the ten electrons that fill the $4 \mathrm{~d}$ shell [41]. Electron exchange between the $4 \mathrm{~d}$ and $5 \mathrm{p}$ shells during photoionization caused the observed increase in the photoionization cross section of the higher lying $5 \mathrm{p}$ state. Coming back to our measurements, it is remarkable that multi-electron correlations also take place during the time-reversed photo-recombination step in HHG. Our observation was confirmed by strong field approximation calculations in which a Coulomb interaction of the returning continuum electron (tunnel ionized from the highest $5 \mathrm{p}$ orbital) with the lower lying $4 \mathrm{~d}$ shell enables energy exchange during the recombination step [31]. Table 1 summarizes the experimental finding of figure 5. Measured and calculated cut-off photon energies are in good agreement from 30 to $160 \mathrm{eV}$ for all laser wavelengths and pulse durations.

It is important to consider limiting factors when aiming for a cut-off at high photon energy. We restrict the following discussion to the single atom response, i.e., neglecting all propagation phenomena and re-absorption of the medium. Obviously, the ultimate limit for an intensity increase is reached when the sample is completely ionized. From table 1, it can be seen that to further increase the saturation intensity one has to reduce the number of cycles during which ionization can deplete the neutral ground state before the maximum of the electric field. At very high intensities, however, different mechanisms like ionization gating can be beneficial for the generation of isolated attosecond pulses [42]. As demonstrated in figure 5, the cut-off extends proportional to the square of the driving laser wavelength; however, one has to pay the price of strong reduction of efficiency [36, 43]. Finally, the cut-off law is halted when the photoionization cross-section of the atom used for HHG vanishes. Because of this, HHG in xenon driven by the sub-two-cycle pulse does not follow the cut-off law anymore even if intensity is further increased (data not shown). As can be seen from the smooth spectral curve obtained with the sub-two-cycle pulse (magenta in figure 5), the entire spectrum is smooth for acquisition conditions comparable to the case of the long pulse (red curve). The spectrum is smooth not only in the cut-off region above $100 \mathrm{eV}$ but also below-in an area where 
the XUV spectrometer is capable of resolving individual harmonics when driven by multi-cycle pulses. This indicates that propagation effects like non-adiabatic sub-cycle dynamics might take place as discussed in [44]. The spectral FWHM of the magenta curve in figure 5 is about $30 \mathrm{eV}$ centred 95 $\mathrm{eV}$ and corresponds to a TL pulse duration of 23 attoseconds which is close to the temporal atomic unit (24.2 attoseconds). This corresponds to the classical time it takes for the electron of a hydrogen atom to travel along one atomic unit of distance (1 atomic unit $=0.529 \AA$ ).

\section{Conclusion}

Multi-cycle OPA pulses are spectrally broadened in a hollowcore fibre and can be compressed with $\mathrm{CM}$ around $1.4 \mu \mathrm{m}$ and solely with bulk material around $1.8 \mu \mathrm{m}$ or longer wavelengths. This approach was successfully demonstrated on two completely different laser and OPA systems, leading to 1.6- and 1.8-cycle Idler pulses, respectively. It confirms the robustness of our approach which is based on available commercial technologies. Passive CEP stabilization was demonstrated with white light seeded OPAs. Wavelength and bandwidth-independent CEP control was carried out by shifting the phase between white light and pump beam before the DFG in the OPA. By applying tunable IR pulses for HHG, we verified experimentally that the cut-off increases proportional to the square of the driving laser wavelength. Furthermore, we show that saturation intensities at a given wavelength can be increased when driving HHG with fewcycle pulses. Finally, 1.8-cycle pulses at $1.8 \mu \mathrm{m}$ wavelength enabled cut-off extension in xenon up to $160 \mathrm{eV}$, enabling for the first time observation of multi-electron correlation effects in $\mathrm{HHG}$ around $100 \mathrm{eV}$, in particular the giant resonance in xenon.

\section{Acknowledgments}

The authors acknowledge the financial support from the Canada Foundation for Innovation (CFI), the Natural Sciences and Engineering Research Council of Canada (NSERC), Le Fonds Québécois de la Recherche sur la Nature et les Technologies (FQRNT) and the Canadian Institute for Photonic Innovations (CIPI).

\section{References}

[1] Fork R L, Brito Cruz C H, Becker P C and Shank C V 1987 Opt. Lett. 12483

[2] Mathies R A, Brito Cruz C H, Pollard W T and Shank C V 1988 Science 240777

[3] Becker P C, Fragnito H L, Brito Cruz C H, Fork R L, Cunningham J E, Henry J E and Shank C V 1988 Phys. Rev. Lett. 611647

[4] Becker P C, Fork R L, Brito Cruz C H, Gordon J P and Shank C V 1988 Phys. Rev. Lett. 602462

[5] Fragnito H L, Bigot J-Y, Becker P C and Shank C V 1989 Chem. Phys. Lett. 160101

[6] Nisoli M, De Silvestri S and Svelto O 1996 Appl. Phys. Lett. 682793
[7] Apolonski A, Poppe A, Tempea G, Spielmann C, Udem Th, Holzwarth R, Hänsch Th W and Krausz F 2000 Controlling the phase evolution of few-cycle light pulses Phys. Rev. Lett. 85740

[8] Jones D J, Diddams S A, Ranka J K, Stentz A, Windeler R S, Hall J L and Cundiff S T 2000 Carrier-envelope phase control of femtosecond mode-locked lasers and direct optical frequency synthesis Science $\mathbf{2 8 8} 635$

[9] Hentschel M, Kienberger R, Spielmann C, Reider G A, Milosevic N, Brabec T, Corkum P, Heinzmann U, Drescher M and Krausz F 2001 Attosecond metrology Nature $\mathbf{4 1 4} 509$

[10] Schultze M et al 2010 Delay in photoemission Science 3281658

[11] Eckle P, Pfeiffer A, Cirelli C, Staudte A, Dörner R, Muller H G, Büttiker M and Keller U 2008 Attosecond ionization and tunneling delay time measurements Science 3221525

[12] Goulielmakis E et al 2010 Real-time observation of valence electron motion Nature 466739

[13] Sansone G et al 2010 Electron localization following attosecond molecular photoionization Nature $\mathbf{4 6 5} 763$

[14] Cavalieri A L et al 2007 Attosecond spectroscopy in condensed matter Nature 4491029

[15] Cavalieri A et al 2007 Intense 1.5-cycle near infrared laser waveforms and their use for the generation of ultrabroadband soft-x-ray harmonic continua New J. Phys. 9242

[16] Goulielmakis E et al 2008 Single-cycle nonlinear optics Science 3201614

[17] Corkum P B and Krausz F 2007 Attosecond science Nature Phys. 3381

[18] Takahashi E J, Kanai T, Ishikawa K L, Nabakawa Y and Midorikawa K 2008 Coherent water window X-ray by phase-matched high-order harmonics Phys. Rev. Lett. 101253901

[19] Shan B and Chang Z 2001 Dramatic extension of the high-order harmonic cut-off by using a long-wavelength driving field Phys. Rev. A 65011804

[20] Popmintchev T, Chen M C, Bahabad A, Gerrity M, Sidorenko P, Cohen P, Christov P, Murnane M M and Kapteyn H C 2009 Phase matching of high harmonic generation in the soft and hard $\mathrm{x}$-ray regions of the spectrum Proc. Natl Acad. Sci. 10610516

[21] Chen M-C, Arpin P, Popmintchev T, Gerrity M, Zhang B, Seaberg M, Popmintchev D, Murnane M M and Kapteyn H C 2010 Bright, coherent, ultrafast soft x-ray harmonics spanning the water window from a tabletop light source Phys. Rev. Lett. 105173901

[22] Corkum P B 1993 Plasma perspective on strong field multiphoton ionization Phys. Rev. Lett. 711994

[23] Gu X et al 2007 Generation of carrier-envelope-phase-stable 2-cycle $740-\mu \mathrm{J}$ pulses at $2.1-\mu \mathrm{m}$ carrier wavelength $O p t$. Exp. 1762

[24] Hong K-H, Huang S-W, Moses J, Fu X, Lai C-J, Cirmi G, Sell A, Granados E, Keathley P and Kärtner F X 2011 High-energy, phase-stable, ultrabroadband $\mathrm{kHz}$ OPCPA at $2.1 \mu \mathrm{m}$ pumped by a picosecond cryogenic Yb:YAG laser Opt. Exp. 1915538

[25] Hauri C P et al 2007 Intense self-compressed, self-phase-stabilized few-cycle pulses at $2 \mu \mathrm{m}$ from an optical filament Opt. Lett. 32868

[26] Mücke O D, Ališauskas S, Verhoef A J, Pugžlys A, Baltuška A, Smilgevičius V, Pocius J, Giniūnas L, Danielius R and Forget L 2009 Self-compression of millijoule $1.5 \mu \mathrm{m}$ pulses Opt. Lett. 342498

[27] Vozzi C, Calegari F, Benedetti E, Gasilov S, Sansone G, Cerullo G, Nisoli M, De Silvestri C and Stagira C 2007 Millijoule-level phase-stabilized few-optical-cycle infrared parametric source Opt. Lett. 322957 
[28] Schmidt B E et al 2010 Compression of 1.8 micron laser pulses to sub two optical cycles with bulk material Appl. Phys. Lett. 96121109

[29] Béjot P, Schmidt B E, Kasparian J, Wolf J-P and Légaré F 2010 Mechanism of hollow-core-fiber infrared supercontinuum compression with bulk material Phys. Rev. A 81063828

[30] Schmidt B E, Shiner A D, Lassonde P, Kieffer J-C, Corkum P B, Villeneuve D M and Légaré F 2011 CEP stable 1.6 cycle laser pulses at $1.8 \mu \mathrm{m}$ Opt. Exp. 196858

[31] Shiner A D, Schmidt B E, Trallero-Herrero C, Wörner H J, Patchkovskii S, Corkum P B, Kieffer J C, Légaré F and Villeneuve D M 2011 Nature Phys. 7464

[32] Baltuška A, Fuji T and Kobayashi A 2002 Controlling the carrier-envelope phase of ultrashort light pulses with optical parametric amplifiers Phys. Rev. Lett. 88133901

[33] Giguère M, Schmidt B E, Shiner A D, Houle M-A, Bandulet H C, Tempea G, Villeneuve D M, Kieffer J-C and Légaré F 2009 Pulse compression of submillijoule few-optical-cycle infrared laser pulses using chirped mirrors Opt. Lett. 341894

[34] Li C, Wang D, Song L, Liu J, Liu P, Xu C, Leng Y, Li R and $\mathrm{Xu} Z 2011$ Generation of carrier-envelope phase stabilized intense 1.5 cycle pulses at $1.75 \mu \mathrm{m}$ Opt. Exp. 196783

[35] Yudin G L and Ivanov M Y 2001 Phys. Rev. A 64013409

[36] Shiner A D et al 2009 Wavelength scaling of high harmonic generation efficiency Phys. Rev. Lett. 103073902
[37] Chowdhuri M B et al 2007 Spectroscopic comparison between 1200 grooves/mm ruled and holographic gratings of a flat-field spectrometer and its absolute sensitivity calibration using bremsstrahlung continuum Rev. Sci. Instrum. 78023501

[38] Yamazaki T et al 1999 Comparison of mechanically ruled versus holographically varied line-spacing gratings for a soft-x-ray flat-field spectrograph Appl. Opt. 384001

[39] Thai A, Hemmer M, Bates P K, Chalus O and Biegert J 2011 Sub-250-mrad, passively carrier-envelope-phase-stable mid-infrared OPCPA source at high repetition rate Opt. Lett. 363918

[40] Becker U et al 1989 Subshell photoionization of Xe between 40 and $1000 \mathrm{eV}$ Phys. Rev. A 393902

[41] Kutzner M, Radojević V and Kelly H P 1989 Extended photoionization calculations for xenon Phys. Rev. A 405052

[42] Ferrari F, Calegari F, Lucchini M, Vozzi C, Stagira S, Sansone G and Nisoli M 2010 High-energy isolated attosecond pulses generated by above-saturation few-cycle fields Nature Photonics 4875

[43] Tate J, Auguste T, Muller H G, Salières P, Agostini P and DiMauro L F 2007 Scaling of wave-packet dynamics in an intense midinfrared field Phys. Rev. Lett. 98013901

[44] Jin C, Le A-T, Trallero-Herrero C A and Lin C D 2011 Generation of isolated attosecond pulses in the far field by spatial filtering with an intense few-cycle mid-infrared laser Phys. Rev. A 84043411 